Keren Zhou, MD

Department of Endocrinology, Diabetes, and Metabolism,

Endocrinology \& Metabolism Institute, Cleveland Clinic
Mohammed J. Al-Jaghbeer, MD

Department of Critical Care Medicine,

Respiratory Institute, Cleveland Clinic
M. Cecilia Lansang, MD, MPH

Department of Endocrinology, Diabetes, and Metabolism,

Endocrinology \& Metabolism Institute, Cleveland Clinic

\title{
Hyperglycemia management in hospitalized patients with COVID-19
}

\section{Posted July 6, 2020}

\section{ABSTRACT}

It has been well established that patients with diabetes who have COVID-19 have a more severe disease course and higher mortality. Providing adequate care for these patients has required hospitals to adapt protocols for monitoring blood glucose and administering therapy to protect both patient and caregiver safety. Inpatient use of continuous glucose monitoring systems or home-use glucose monitoring systems has provided options for reduced contact glucose monitoring. For therapy, protocols for managing hyperglycemia and diabetes ketoacidosis have been designed with less frequent monitoring and medication administration. Finally, telemedicine has allowed for consultative care in a manner not requiring physical proximity.

\section{BACKGROUND}

As healthcare systems respond to the COVID-19 pandemic, it has become increasingly clear that patients with pre-existing diabetes mellitus (DM) are more likely to be hospitalized with complications of COVID-19. Studies from Wuhan, China, show a significant percentage of COVID-19-positive patients had DM, and DM was significantly more common in those admitted to the intensive care unit (ICU) and among fatalities (26.9\% vs $6.1 \%) .1,2$ Beyond the prevalence of DM in patients with COVID-19, there are data from both the severe acute respiratory distress syndrome outbreak and the current COVID-19 pandemic showing that inpatient hyperglycemia is associated with worse outcomes. ${ }^{3,4}$

At Cleveland Clinic, we have implemented various COVID-19-related modifications related to the care of patients with hyperglycemia and DM on the regular

The statements and opinions expressed in COVID-19 Curbside Consults are based on experience and the available literature as of the date posted. While we try to regularly update this content, any offered recommendations cannot be substituted for the clinical judgment of clinicians caring for individual patients.

doi:10.3949/ccjm.87a.ccc012 nursing floors and in the ICUs. We will review these changes and what is currently known about the interaction of hyperglycemia, DM, and COVID-19.

\section{DM AND COVID-19 INTERACTIONS: CURRENT UNDERSTANDING}

Diabetes and hyperglycemia are widely recognized to be associated with increased likelihood of a more severe course of COVID-19., 1,2,-6 In patients with DM, those of older age ( $>55$ years), with pre-existing hypertension, lower albumin levels, and higher C-reactive protein on admission did worse during their hospital course, ${ }^{5,7}$ as did patients on insulin. ${ }^{7}$ These worse outcomes are seen both in patients with DM prior to admission and in those with more significant hyperglycemia while in the hospital. ${ }^{4}$ As has been noted in non-COVID-19-related studies, hospitalized patients with COVID-19 and hyperglycemia without known DM actually have worse outcomes than those with hyperglycemia and known DM, likely related to the fact that the hyperglycemia in patients without known DM represents a more aggressive infection or reaction with COVID-19.4

\section{MANAGEMENT OF HYPERGLYCEMIA ON REGULAR NURSING FLOORS}

A key part of managing patients with COVID-19 is protecting caregivers. Personal protective equipment (PPE) plays a crucial role in this. Shortages of PPE during this pandemic are well documented, and efforts to preserve PPE have led to modifications in management from monitoring, therapeutic, and consultative standpoints.

Prior to this pandemic, 2 continuous glucose monitoring systems (CGMS) had been FDA-approved for hospital use, accessing blood samples through a central venous catheter or a peripheral vein (OptiScanner 5000 and GlucoScout). ${ }^{8}$ These are suitable for use in the ICU but are often not available, as is the case at 
our institution. On the regular nursing floors, subcutaneously applied commercial CGMSs had previously lacked FDA approval for inpatient use. However, at the beginning of April 2020, the FDA announced that it would not object to the use of the FreeStyle Libre 14 day or Dexcom CGMS for inpatient use during the COVID-19 pandemic. ${ }^{9,10}$ In addition, Abbott Laboratories (maker of the FreeStyle Libre CGMS) and Dexcom are donating 25,000 sensors and 10,000 phones/sensors, respectively, for hospital use. Hospitals now have the ability to harness this technology for blood glucose monitoring on regular nursing floors, bearing in mind precautions on when to check with a point-of-care blood glucose meter, such as when the clinical picture does not match the readings from the CGMS. These CGMS are not for use in critically ill patients. ${ }^{11}$

The use of CGMS on regular nursing floors presents some inherent challenges. Nurses on COVID19-designated units will have to receive additional instruction, and modifications of nursing policy surrounding glucose monitoring would have to be addressed. Ideally, a system akin to glucose telemetryy $^{12}$ could be used, but it will take some time to develop and implement and may lead to additional alarm fatigue. Without telemetry-like capabilities, the CGMS will be used mainly for replacement of the usual 4 or more fingersticks needed for point-of-care blood glucose readings. Nurses would still need to see the reading and upload this on our electronic record, but the rest of the point-of-care blood glucose process is eliminated.

There is concern that the additional data from CGMSs, while ideal for catching impending extremes of blood glucose levels, may lead to micromanagement of blood glucose, placing additional burden on nursing and PPE use. Whether this proves to be a real issue, however, is a theoretical concern at this point. Providers also will need to be aware that CGMSs should be removed during certain procedures such as CT scans and MRI, which may lead to a waste of sensors. In addition, if supply is limited, we will need to be more discerning with inpatient selection. Intuition suggests that patients who are on multiple daily insulin injections would be the priority; however, patients on oral therapy may benefit because CGMS can detect rising blood glucose without multiple daily fingersticks.

For those patients comfortable and able to do point-of-care blood glucose checks, the FDA now allows home-use glucose meters in the hospital. ${ }^{13}$ This applies to patients' own meters or home-use meters dispensed by hospitals for use by a single patient. Permitting patients to use their own meters may provide them with a sense of comfort, and it reduces the need for costly hospital-approved devices that have to be sanitized between patients by nursing personnel. However, providing glucose meters to patients allows for uniform training of nursing personnel as well as addressing the needs of patients who do not bring their glucose meters.

Insulin remains the primary treatment for hyperglycemia in hospitalized patients. ${ }^{14}$ However, certain considerations can help minimize caregiver exposure and PPE use. First, avoidance of sliding scale insulinalone regimens would help reduce need for frequent insulin administrations. In addition, using oral agents that have renal dosing and low risk of hypoglycemia, such as sitagliptin, a dipeptidyl peptidase-4 (DPP-4) inhibitor, in mildly hyperglycemic patients is another strategy to reduce blood glucose checks if a CGMS is not available. ${ }^{15}$

There are ongoing conversations concerning the role of DPP-4 inhibitors in COVID-19 cases as evidence suggests that the COVID-19 surface glycoprotein-mediating host cell entry has the ability to interact with DPP $-4 .{ }^{16}$ Although DPP-4 inhibitors do not appear to bind to the same site as the virus, DPP-4 has become a potential therapeutic target, and we have more to learn about how the use of DPP-4 inhibitors impacts the likelihood and course of COVID. 19. Another category of DM medications that has received additional scrutiny is the sodium-glucose cotransporter-2 (SGLT-2) inhibitors. If there is any indication of COVID-19 in a patient on an SGLT-2 inhibitor, that therapy should be discontinued as there is risk for euglycemic diabetic ketoacidosis. ${ }^{17}$

\section{MANAGEMENT OF HYPERGLYCEMIA IN ICU}

Hyperglycemia is common in ICU patients and is associated with worse outcomes. ${ }^{18}$ In a small series of 15 patients with diabetic ketoacidosis and COVID19, one-third died. ${ }^{19}$ There is increased risk of diabetic ketoacidosis in patients with COVID-19. Our protocols (and many others) for diabetic ketoacidosis intravenous (IV) insulin infusion require hourly blood glucose monitoring and titration based on a dosage calculator built into our electronic medical record. COVID-19-positive patients whose diabetic ketoacidosis parameters improve while on the protocol are changed to our less aggressive hyperglycemia IV insulin infusion protocol, conventionally used for nondiabetic ketoacidosis patients in the ICUs, with blood glucose monitoring every 2 to 3 hours. 
Our hyperglycemia IV insulin infusion protocol has retrospectively demonstrated the same resolution time with no difference in the frequency of hypoglycemia as our diabetic ketoacidosis protocol, ${ }^{20}$ and it can be adopted for use in patients with mild to moderate diabetic ketoacidosis. A protocol using blood glucose monitoring every 2 hours fits with the strategy of bundling care for patients with COVID-19 in the ICUs to every 2 hours to protect caregivers and conserve PPE. Another option is to place the drips requiring frequent titration to a location outside the patient room (Figure 1).

A subcutaneous treatment protocol for diabetic ketoacidosis can be adopted and modified from other institutions, but these protocols use monitoring every hour or every other hour. ${ }^{21,22}$ In addition, adopting a subcutaneous treatment protocol would require significant work-flow changes for our providers who are already adapting to the ever-changing patient care needs as the understanding of COVID-19 evolves.

\section{CONSULTATIONS IN COVID-19 ERA}

To protect caregivers and preserve PPE, we have adopted outpatient telehealth practices for use in inpatient endocrinology consults: e-consult, telephone, and virtual consults. The primary difference is in the mode of information gathering. The e-consults replace what are called curbside consults. In an e-consult, caregivers review the electronic medical record but have no direct contact with patients. The telephone and virtual consults represent differences in the contact with the patient-via telephone (audio only) or virtually (both audio and visual). We have conducted patient visits by using the patient's personal smart phone or tablet or using communal iPads and InTouch Health devices (similar to tablets) or by using the telephone assigned to the patient's bed space on our COVID-19 units. Inpatient blood glucose management has always represented an opportunity for telehealth management decisions that rely heavily on data in the electronic medical record (eg, point-of-care blood glucose, medication administration).

Inpatient diabetes education consults have traditionally been done during in-person bedside visits by the diabetes care and education specialist. Before the pandemic, educational materials were available on our Cleveland Clinic website, including instructional videos for administering insulin and using blood glucose meters correctly, as well as an illustrated diabetes booklet focusing on survival skills. Diabetes care and education specialists can direct patients to the online

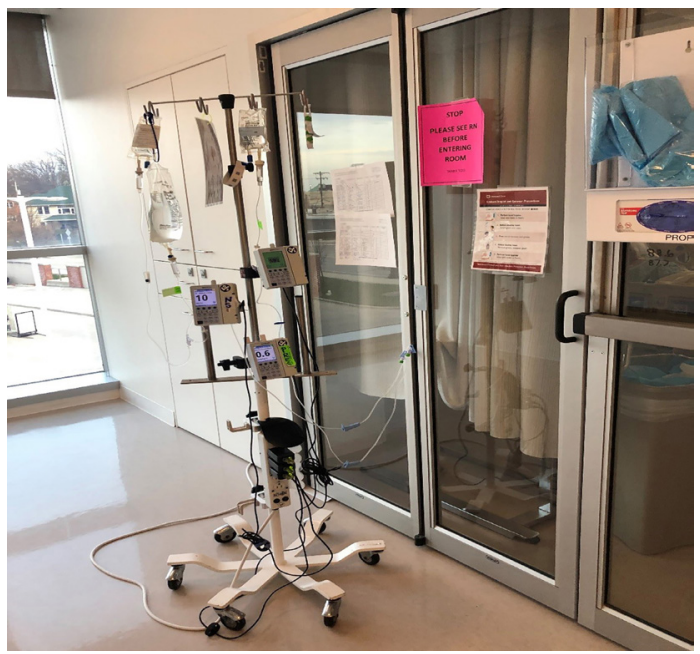

Figure 1. Pole for maintaining IV medications outside a patient's room.

materials during virtual education sessions.

\section{TAKE-HOME POINTS}

The COVID-19 pandemic has driven us to rethink how to manage our patients within safe parameters while conserving PPE and protecting caregivers. As more data become available, we can reprogram to adapt to the changing evidence. For now, we recommend the following when managing hyperglycemia in the critically ill patient with COVID-19:

- Diabetes is a well-documented risk factor for COVID-19 severity.

- For monitoring blood glucose, when deciding the frequency of glucose checks, be aware of staff safety and PPE need as well as patient safety. Striking a balance requires reliance on clinical judgment as protocols are modified to adapt to this pandemic. Consider use of continuous glucose monitoring systems, as appropriate.

- Consider alternative protocols for management of hyperglycemia and diabetes ketoacidosis, including use of protocols with less frequent monitoring and medication administration.

- Consider an endocrinology consult, even by telemedicine, to help manage blood glucose if there is risk of significant patient instability.

\section{REFERENCES}

1. Guan W, Ni Z, Hu Y, et al. Clinical characteristics of coronavirus disease 2019 in China. N Engl J Med 2020; 382:1708-1720. doi. org/10.1056/NEJMoa2002032

2. Yang $X, Y u Y, X u J$, et al. Clinical course and outcomes of critically ill patients with SARS-CoV-2 pneumonia in Wuhan, China: a singlecentered, retrospective, observational study. Lancet 2020. doi. 
org/10.1016/ S2213-2600(20)30079-5

3. Yang JK, Feng Y, Yuan MY, et al. Plasma glucose levels and diabetes are independent predictors for mortality and morbidity in patients with SARS. Diabet Med 2006; 23(6):623-628. doi:10.1111/j.1464-5491.2006.01861.x

4. Bode B, Garrett V, Messler J, et al. Glycemic characteristics and clinical outcomes of COVID-19 patients hospitalized in the United States. J Diabetes Sci Technol 2020; May 9. doi: 10.1177/1932296820924469

5. Huang I, Lim MA, Pranata R. Diabetes mellitus is associated with increased mortality and severity of disease in COVID-19 pneumonia - a systematic review, meta-analysis, and meta-regression. Diabetes Metab Syndr 2020; 14(4):395-403. doi: 10.1016/j.dsx.2020.04.018

6. Riddle MC, Buse JB, Franks PW, et al. COVID-19 in people with diabetes: urgently needed lessons from early reports. Diab Care 2020; 43(7):1378-1381. doi: 10.2337/dci20-0024

7. Chen Y, Yang D, Cheng B, et al. Clinical characteristics and outcomes of patients with diabetes and COVID-19 in association with glucoselowering medication. Diabetes Care 2020; 43(7):1399-1407. doi. org/10.2337/dc20-0660

8. Umpierrez GE, Klonoff DC. Diabetes technology update: use of insulin pumps and continuous glucose monitoring in the hospital. Diabetes Care 2018; 41(8):1579-1589. doi: 10.2337/dci18-0002

9. Abbott. Abbott's FreeStyle ${ }^{\circledR}$ Libre 14 day system now available in U.S. for hospitalized patients with diabetes during COVID-19 pandemic. https://abbott.mediaroom.com/2020-04-08-Abbotts-FreeStyle-R-Libre-14-Day-System-Now-Available-in-U-S-for-Hospitalized-Patients-with-Diabetes-During-COVID-19-Pandemic. Accessed July 1, 2020.

10. Dexcom Inc. Fact sheet for healthcare providers: use of Dexcom continuous glucose monitoring systems during the COVID-19 pandemic. https://www.dexcom.com/hospitalfacts. Accessed July 1, 2020.

11. Wallia A, Umpierrez GE, Rushakoff RJ, et al. Consensus statement on inpatient use of continuous glucose monitoring. J Diabetes Sci Technol 2017; 11(5):1036-1044. doi: 10.1177/1932296817706151

12. Spanakis EK, Levitt DL, Siddiqui T, et al. The effect of continuous glucose monitoring in preventing inpatient hypoglycemia in general wards: the glucose telemetry system. J Diabetes Sci Technol 2018; 12(1):20-25. doi: 10.1177/1932296817748964

13. U.S. Food and Drug Administration. FAQs on home-use blood glucose meters utilized within hospitals during the COVID-19 pandemic. https://www.fda.gov/medical-devices/blood-glucosemonitoring-devices/faqs-home-use-blood-glucose-meters-utilizedwithin-hospitals-during-covid-19-pandemic. Accessed July 1, 2020.

14. American Diabetes Association. Diabetes care in the hospital: standards of medical care in diabetes-2020. Diabetes Care 2020; 43(Suppl 1):S193-S202. doi: 10.2337/dc20-S015

15. Umpierrez GE, Gianchandani R, Smiley D, et al. Safety and efficacy of sitagliptin therapy for the inpatient management of general medicine and surgery patients with type 2 diabetes: a pilot, randomized, controlled study. Diabetes Care 2013; 36(11):3430-3435. doi: $10.2337 / \mathrm{dc} 13-0277$

16. Bassendine MF, Bridge SH, McCaughan GW, Gorell MD. COVID囚19 and comorbidities: a role for dipeptidyl peptidase 4 (DPP4) in disease severity? J Diabetes 2020; May 11. doi: 10.1111/1753-0407.13052

17. Rosenstock J, Ferrannini E. Euglycemic diabetic ketoacidosis: a predictable, detectable, and preventable safety concern with SGLT2 inhibitors. Diabetes Care 2015; 38(9):1638-1642. doi: 10.2337/ dc15-1380

18. Krinsley JS. Association between hyperglycemia and increased hospital mortality in a heterogeneous population of critically ill patients. Mayo Clin Proc 2003; 78(12):1471-1478. doi:10.4065/78.12.1471

19. Li J, Wang X, Chen J, et al. COVID-19 infection may cause ketosis and ketoacidosis. Diabetes Obes Metab 2020; Apr 20. doi: 10.1111/ dom. 14057

20. Chen A, Ketz J, Reddy A, et al. Comparison of two different insulin nomograms for treatment of patients with diabetic ketoacidosis. Crit Care Med 2018; 46(Suppl 1):203. doi: 10.1097/01. ccm.0000528457.23498.c5
21. Kitabchi AE, Umpierrez GE, Miles JM, et al. Hyperglycemic crises in adult patients with diabetes. Diabetes Care 2009; 32(7):1335-1343. doi: $10.2337 /$ dc09-9032

22. Umpierrez GE, Cuervo R, Karabell A, Latif K, Freire AX, Kitabchi AE. Treatment of diabetic ketoacidosis with subcutaneous insulin aspart. Diabetes Care 2004; 27(8):1873-1878. doi: 10.2337/ diacare.27.8.1873

Correspondence: M. Cecilia Lansang, MD, MPH, Department of Endocrinology, Diabetes and Metabolism, Endocrinology \& Metabolism Institute, F20, Cleveland Clinic, 9500 Euclid Avenue, Cleveland, $\mathrm{OH}$ 44195; lansanm@ccf.org 